\title{
Is it important to investigate the clinical periodontal and microbiolog- ical condition in juvenile idiopathic arthritis patients (JIA)?
}

\section{Consuelo Romero- Sánchez ${ }^{1,2 *}$}

Pediatric Rheumatology Program, School of Medicine, Unit of Oral Basic Investigation, School of Dentistry, Universidad El Bosque, Bogota, Colombia. ${ }^{2}$ Group of Applied Clinical Immunology, Rheumatology and Immunology Department Hospital Militar Central, School of Medicine, Universidad Militar Nueva Granada, Bogota, Colombia.

\section{Article Info}

\section{Article Notes}

Received: October 05, 2017

Accepted: November 24, 2017

\section{*Correspondence:}

Dr. Consuelo Romero- Sánchez, Group of Applied Clinical Immunology, Rheumatology and Immunology Department Hospital Militar Central, School of Medicine, Universidad Militar Nueva Granada, Bogota, Colombia; E-Mail: romeromaria@unbosque.edu.co

C 2017 Romero- Sánchez C. This article is distributed under the terms of the Creative Commons Attribution 4.0 International License.

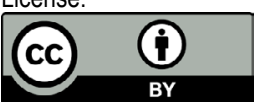

Juvenile idiopathic arthritis (JIA), is the most frequent chronic inflammatory arthropathy in pediatric patients ${ }^{1}$. It is characterized by the presence of arthritis before the age of 16, lasting more than 6 weeks and of unknown etiology, after excluding others causes. The joint involvement is made manifest by joint edema, movement limitation, joint pain or local heat sensation. This syndrome includes different categories that all share the presence of arthritis. Seven different clinical phenotypes exist which allow for the establishment of homogeneous groups regarding its beginning, course, and prediction that are mutually exclusive $\mathrm{e}^{2,3}$.

The cause ofJIA remains unknown. It is considered a multifactorial etiology disease. It is known that genetic predisposition of the host and environmental influences, such infections that can act as pathogenic process inductors, play a role ${ }^{4,5}$.

The category of JIA, represented by polyarthritis and rheumatoid factor positive patients, is characterized by having a symmetric involvement in small joints, erosive, beginning mostly in teenage girls. These represent $5 \%$ of JIA patients and they share clinical and immunogenetic characteristics with adult RA patients. The rheumatoid factor positive (RF) polyarticular JIA patients, usually reach adulthood with the active disease and develop functional disability ${ }^{5}$. The formation of anti-citrullinated protein auto-antibody (ACPAs) has been identified in this JIA subtype, but less frequently compared to adults with rheumatoid arthritis (RA). The association of Rheumatoid Arthritis (RA) with periodontal disease (PD) has been previously described in adults, and periodontal treatment reduces markers of RA activity ${ }^{6}$.

PD is a chronic infection that often affects adults, consisting of a destruction of the supporting tissues of the tooth as an immune inflammatory response of the host. Children and teenagers can develop it. The disease is related to anaerobic Gram-negative bacilli inside the gingival sulcus, mainly associated with Porphyromonas gingivalis (P.gingivalis) ${ }^{7}$. This microorganism expresses the enzyme peptidyl arginine deiminase, similar to that involved in the pathogenesis of RA, and can increase the levels of ACPAs in adults with periodontitis ${ }^{8}$.

It is clinically manifested by an inflamed gingiva, with the presence of periodontal pockets or as a gingival recession, due to attachment 
loss and bone destruction. There is not much information about the relationship between periodontal conditions and children with JIA, though literature has suggested that JIA patients are susceptible to having periodontal disease ${ }^{7}$.

It is important to insist on the relationship between JIA and periodontal disease in the medium and long term, since the transmission of periodontal pathogenic bacteria can be transmitted between family members, more specifically to children from their parents ${ }^{9}$. Recent reports have pointed to the association between JIA and oral diseases such as tooth decay, periodontal disease, salivary structure, temporomandibular jaw joint and alterations in the development of facial complex, showing that systematic effects of the disease can affect oral health ${ }^{10,11}$.

We conducted a study of JIA in 54 patients according to the International League of Associations for Rheumatology criteria (including the 7 categories). We excluded children under the age of 10 , those with recent use of antibiotics, orthodontic treatment and those with fewer than 6 teeth. All were given a clinical periodontal exam. The indices used for diagnosis were as follows: pocket

depth, clinical attachment loss, bleeding on probing and the plaque index. Microbiological analysis and antibodies against bacteria related to this entity were also analyzed. We found that the percentage with high levels of plaque, bleeding and swelling was considerable, and an important percentage of this group was diagnosed as periodontitis cases. On the other hand, the microbiological analysis indicated that $23.5 \%$ of patients had P. gingivalis in subgingival plaque. The presence of $P$. gingivalis was independent of age, the age of onset of symptoms, and bacteria related to periodontal disease of the adult. Moreover, antibodies IgG1 directed against P.gingivalis (ATCC 33277 and W83 strains) were associated with RF autoantibodies ${ }^{12}$, and all patients with ACPAs positives had higher IgG1 against $P$. gingivalis ${ }^{13}$. These preliminary results show that JIA patients in treatment may have periodontitis and related bacteria. Therefore, it is important to continue to investigate the periodontal condition of JIA patients and their family environments to prevent more severe outcomes in adulthood.

\section{References}

1. Prakken B, Albani S, Martini A. Juvenile idiopathic arthritis. Lancet. 2011; 2138-49.

2. Prahalad S. Genetics of juvenile idiopathic arthritis: an update. Curr Opin Rheumatol. 2004; 16: 588-94.

3. Petty RE, Southwood TR, Baum J et al. Revision of the proposed classification criteria for juvenile idiopathic arthritis: Durban, 1997. J Rheumatol. 1998; 1991-4.

4. Kugathasan S, Judd RH, Hoffmann RG, et al.1. Epidemiologic and clinical characteristics of children with newly diagnosed inflammatory bowel disease in Wisconsin: a statewide population-based study. J Pediatr. 2003; 525-3.

5. Gowdie PJ, Tse SML. Juvenile Idiopathic Arthritis. Pediatr Clin N Am. 2012; (59): 301-27.

6. Bello-Gualtero JM, Lafaurie GI, Hoyos LX, et al. Periodontal disease in individuals with a genetic risk of developing arthritis and early rheumatoid arthritis: a cross-sectional study. J Periodontol. 2016; 87: 346-356.

7. Pihlstrom BL, Michalowicz BS,Johnson NW. Periodontal diseases. Lancet. 2005; 366: 1809-20.

8. Mikuls TR, Payne JB, Yu F, et al. Periodontitis and Porphyromonas gingivalis in Patients With Rheumatoid Arthritis. Arthritis Rheum. 2014; 66: 1090-1100.

9. Sirinian G, Shimizu T, Sugar C, et al. Periodontopathic bacteria in young healthy subjects of different ethnic backgrounds in Los Angeles. J Periodontol. 2002 Mar; 73: 283-8.

10. Reichert S, Machulla HKG, Fuchs $C$, et al. Is there a relationship between juvenile idiopathic arthritis and periodontitis. J Clin Periodontol. 2006; 33: 317-23.

11. Ahmed N, Bloch-Zupan A, Murray KJ, et al. Oral health of children with juvenile idiopathic arthritis. J Rheumatol. 2004; 31: 1639-43.

12. Bello-Gualtero JM, Lafaurie GI, Hoyos LX, et al. Periodontal disease in individuals with a genetic risk of developing arthritis and early rheumatoid arthritis: a cross-sectional study. J Periodontol. 2016; 87(4): 346-56.

13. Romero-Sánchez C, Malagón C, Vargas C, et al. Porphyromonas gingivalis and IgG1 and IgG2 Subclass Antibodies in Patients with Juvenile Idiopathic Arthritis. J Dent Child (Chic). 201715; 84: 72-9 